\title{
Assessment of Characteristics and Conditions before the End of Lockdown
}

\author{
David San-Martín-Roldán1", Francisca Rojo-Lazo1, Aracelis Calzadilla-Núñez², Pablo San-Martín-Roldán³,..
}

\author{
${ }^{1}$ Escuela de Obstetricia y Puericultura, Facultad de Medicina, Universidad de Valparaíso, Valparaíso, Chile \\ ${ }^{2}$ Facultad de Ciencias de la Salud, Universidad Autónoma de Chile, Santiago, Chile \\ ${ }^{3}$ Instituto de Nutrición y Tecnología de los Alimentos (INTA), Universidad de Chile, Santiago, Chile
}

\begin{abstract}
After months of blockades and restriction, the decision of the best time to end the lockdown after the first wave of the COVID-19 pandemic is the big question for health rectors. This study aimed to evaluate the characteristics and conditions for ending the blockade after the first wave of COVID-19. Data on the variables of interest were subjected to linear and non-linear regression studies to determine the curve that best explains the data. The coefficient of determination, the standard deviation of $\mathrm{y}$ in $\mathrm{x}$, and the observed curve of the confidence interval were estimated. Regression which was estimated, subsequently revealed the trend curve. The study found that all dependent variables tend to decrease over time in a quadratic fashion, except for the variable for new cases. In general, the $\mathrm{R}^{2}$ and mean absolute percentage error (MAPE) estimated were satisfactory: gradual and cautious steps should be taken before ending the lockdown. The results suggested that surveillance of crucial indicators (e.g., incidence, prevalence, and PCR test positivity) should be maintained before lockdown is terminated. Moreover, the findings indicated that long-term preparations should be made to contain future waves of new cases.
\end{abstract}

Keywords: COVID-19, forecasting, lockdown, SARS-CoV-2

\section{Introduction}

The global population is possibly experiencing the most critical juncture of this millennium due to the emergence of the Corona Virus Disease 2019 (COVID19) (e.g., the disease caused by the coronavirus SARS$\mathrm{CoV}-2)$ pandemic. In the absence of definitive treatment modalities and lack of development of a vaccine, the only effective strategy currently appears to be preventive in nature. Containment and restrictive measures result in considerably low rates of transmission of the virus. Therefore, a significant part of the world's population is at home as a strategy for containing the pandemic. ${ }^{1}$

Following many Asian and European countries, cities are closed to fight the virus. The most stringent social isolation measure taken to date is total closure. This scheme is projected to be the most effective in preventing the spread of the infection, leading to major negative social and economic consequences. However, governments worldwide have declared that the closure is effective, which resulted in extended periods. ${ }^{2}$

Notably, Chile has performed poorly in terms of prevention. In this regard, understanding the dynamics of infection transmission is essential because it could determine whether outbreak control measures are having a significant effect. Many governments have incorporated control measures, such as quarantine, travel restriction, and airport inspection for travelers. However, the effectiveness of such measures in controlling the outbreak is inconclusive. ${ }^{3,4}$

Many European countries implemented unprecedented non-pharmacological interventions, such as school closure and national closure. Such interventions and the lockdown exerted a significant effect on the reduction of the transmission of the virus. Restricting the movement of people and reducing contact can help to contain the pandemic. Patterns of change during lockdown periods indicate effectiveness in slowing the spread of the virus. However, it must maintain a continuous lockdown to control the transmission of SARS-CoV-2. 3,5

Moreover, this pandemic has generated fears of an impending economic crisis and recession. Socialdistancing, self-isolation, and travel restrictions have reduced the workforce in all economical sectors and caused many job losses. Schools have closed, and the need for manufactured goods has decreased. In contrast, medical supplies have increased significantly. In addition,

Correspondence*: David San-Martín-Roldán, Escuela de Obstetricia y Puericultura, Facultad de Medicina, Universidad de Valparaíso, Angamos 655 Reñaca, Viña del Mar, Chile, E-mail: dasanmaro@gmail.com, Phone: +5696 4559167

Received : July 20, 2020

Accepted : January 02, 2021

Published: February 20, 2021 
the food sector has faced increased demands due to panic buying and storage of food products. ${ }^{6}$

After closure, social isolation has led to economic difficulties and adverse psychological reactions, which are often more significant than physical suffering. According to the World Health Organization (WHO), anxiety and distress have been natural psychological responses to the current situation. Stress, anxiety, and depression manifest themselves when people are under life-threatening circumstances and must face the impending problem of death. These conditions have been intensified if families need to be separated due to the uncertainty of disease progression, insufficient supply, financial losses, and increased risk perception, which are typically magnified by incomplete information and inadequate communication through the media in the early phase of the pandemic. ${ }^{7}$

Chile has initiated de-escalation, thus requiring knowledge about the best time for the end of restriction. The study intended to forecast the future of lockdown and its favorable epidemiological conditions by analyzing three elements, such as incidence, prevalence, and polymerase chain reaction (PCR) test positivity. Such elements were vital to the assessment of the progress of the pandemic. Therefore, this information is a prerequisite to the decision-making of policymakers about future strategies.

\section{Method}

Data was collected from the COVID-19 database of the Chilean Ministry of Science, Technology, Knowledge, and Innovation. The data are publicly available and adhere to ethical regulations. ${ }^{8}$

The study estimated the post-peak decline curves for each variable of interest: new cases $(6,938$ as of June 14 , $2020)$, active cases $(37,307$ as of June 21,2020$)$, and positive PCR tests (39.63\% as of June 10, 2020).

Data for the said variables were subjected to linear and non-linear regression studies to determine which curve best explains the data using analysis of sequential variance. The determination coefficient $\left(\mathrm{R}^{2}\right)$, which is the standard deviation of the values of the dependent variable $\mathrm{y}$ with respect to the independent variable $\mathrm{x}$ in each estimated curve $(S y \cdot x)$ was calculated. Additionally, regression estimates were made with a confidence interval $(\mathrm{CI})$ of $1 \%$ error. The variables under the study trends regarding the explanatory variable (time in days) were estimated using linear and quadratic regressions. The errors in the estimation of the curves were calculated using the error indicators mean absolute percentage error (MAPE), mean absolute deviation (MAD), and mean signed deviation (MSD). Mean absolute percentage error (MAPE) enables the estimation of the magnitude (in percent) of the curve error, whereas two MAD and MSD

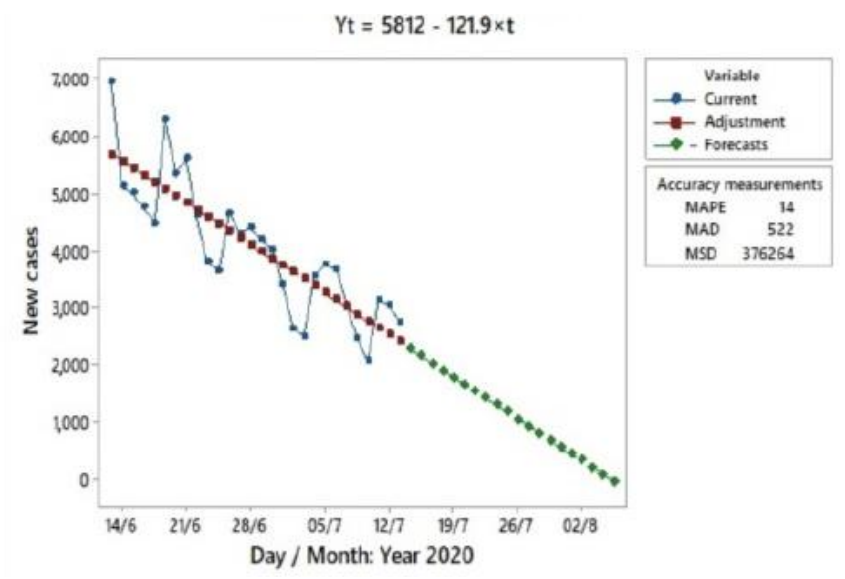

Figure 1. Results of the Trend of Incidence (New Cases) of COVID-19 in Chile at Post-Peak

compare a trend curve with another curve. The lower the indicators are, the better the explanation that the behavior of the data reflects a curve that describes better than others. The program used for the estimates was Minitab 18.0.

\section{Results}

Figure 1 shows the estimation of the trend curve of new cases over time (in days). A linear curve was observed to represent this behavior best. The $\mathrm{R}^{2}(71.9 \%)$ value can be considered relatively high, and this trend curve explains $71.9 \%$ of all existing variations. The value of Sy.x was 643.3. The MAPE value reached $14 \%$, and this magnitude of error explains the estimated $\mathrm{R}^{2}$ value.

Figure 2 depicts the estimation of the active curve of case trend over time (in days). The study observed that a descending quadratic curve best represents this behavior. The $\mathrm{R}^{2}(96 \%)$ value was extremely high, such that this trend curve explains $96 \%$ of all existing variations. The value of $\mathrm{Sy} \cdot \mathrm{x}$ was 774.6 . The MAPE value reached $2 \%$, and this magnitude of error illustrates the magnitude of the estimated $\mathrm{R}^{2}$ value.

Figure 3 displays the results of the estimation of the trend curve of the positive PCR tests (\%) over time (in days). The study observed that a descending quadratic curve best represents this behavior. The $\mathrm{R}^{2}(72.5 \%)$ value was relatively high. Therefore, the trend curve explains $72.5 \%$ of all existing variation. The value of Sy.x was 2.88. The MAPE value reached $7.35 \%$, and this magnitude of error explains the estimated $\mathrm{R}^{2}$ value. The MAD and MSD values can not be compared with other curves because the literature lacks estimates similar to those in the present study.

\section{Discussion}

The mathematical model of any situation aims to describe the essential components and predict certain ge- 


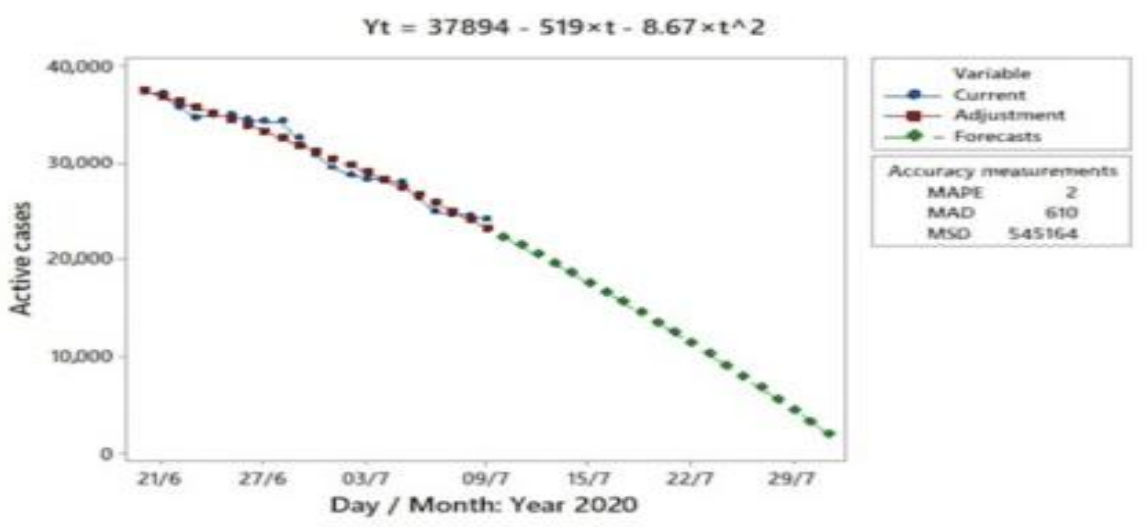

Figure 2. Results of the Trend of Prevalence (Active Cases) of COVID-19 in Chile at Post-Peak

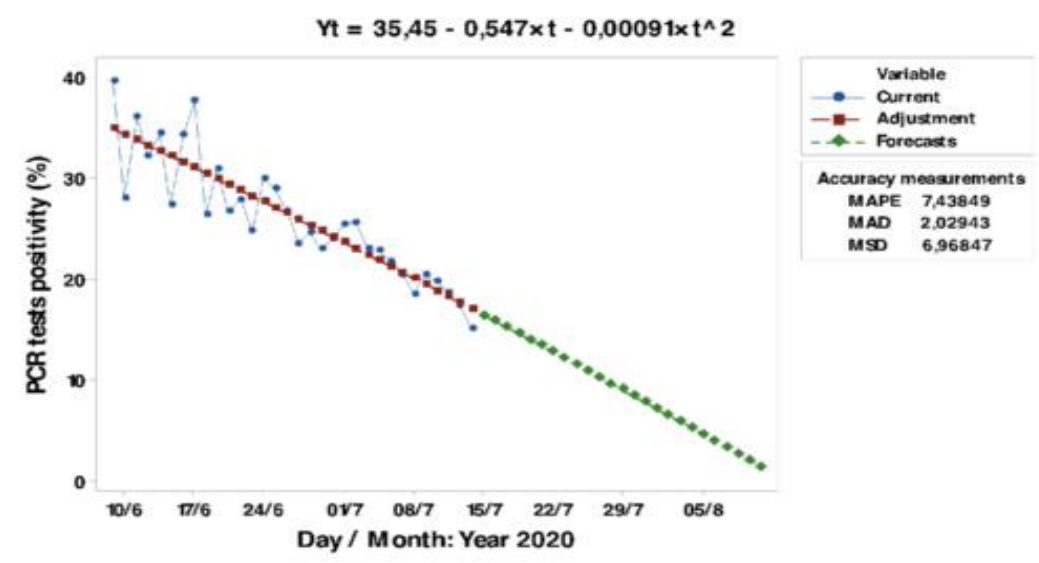

Figure 3. Results of the Trend of Positivity of COVID-19 PCR Tests in Chile, after the PostPeak of Positivity

neral trends. However, an accurate prediction is impossible. Therefore, the proposed model only provides a basis for obtaining a mechanism for understanding under the type of restrictions and current population conditions. One of the epidemiology tasks is predicting the evolution of infectious diseases using mathematical models. However, such models exclude the specific characteristics of the affected population. ${ }^{9}$

The most effective policy is a combination of screening and blocking. Screening reduces the risk of infection, whereas blocking prevents people from returning to normal life prematurely. Notably, the longer the time of home lockdown, the shorter the period of mandatory restrictions required despite the adverse the consequences of the lockdown. For this relationship to be fulfilled, population behavior is essential. Unfortunately, in Chile, this necessary condition has only been partially respected. ${ }^{10}$

Chile has surpassed the minimum barrier of per capita PCR testing, which several countries reported on the date they finished the lockdown. This difference accentuates the perception of Chile's poor performance in terms of prevention of the pandemic. Nevertheless, the deescalation phase should increase the daily PCR tests. The current analysis indicates that the opposite is happening. European,11-20 Asian,21,22 Oceanic, 23,24 and South American countries (Uruguay), ${ }^{25}$ have finished their first major period of lockdown upon reaching the following minimum conditions on average in the context of the COVID-19 pandemic: incidence rate $=1.26(100,000$ in habitants $)$, prevalence rate $=61.05(100,000$ inhabitants), and rate of positive PCR tests $=2.81 \% .26$

According to the abovementioned indicators, the study estimates that the start date for the gradual de-escalation of restrictions should begin after July 30, 2020. The reason for this date is to determine the end date of the containment, thus avoiding the saturation of health services and achieving success by taking into account only the criterion of active cases, which is the closest to being reached. However, evidence shows that beginning the end of mandatory de-escalation on August 3, 2020 would be completely safe and successful given the three com- 
bined indicators (e.g., incidence, prevalence, and positivity). 4

The lockdown should be lifted without risk of overwhelming health services. If all restrictions are universally lifted, it could trigger a rapid resurgence of infections and lead to more deaths. Second and third waves are expected after reopening and a considerable time before the development of any effective vaccine, generation of collective immunity, and final resolution of the pandemic. Second waves are predictable and should be managed in proportion. If a return to containment is necessary for mitigation, then it should occur without generating suspicion. With the decrease in the number of active cases, traceability will become easier and presumably more effective. Thus, preparing for future outbreaks is necessary, especially by strengthening primary care services. Such preparation is precisely the function of primary care and nursing staff, which is crucial to the post-peak COVID19 phase because people do not internalize the social costs of the spread of the virus and opt to return to normal life soon. The estimation indicated $59 \%-85 \%$ of the population should be infected pandemic in the absence of an effective vaccine or treatment. Therefore, the strategy should be managing the spread through phased release measures. Any flexibility in the sudden release of the lockdown or impossibility of achieving contact tracing can lead to exponential transmission, leading to many unmanageable cases given the available health infrastructure and human resources. Simultaneously, evidencebased protocols for possible strategies should be prepared before the end of the lockdown. ${ }^{27-30}$

The study recommends that in the period leading up to the end of the lockdown, teleworking should be intensified, and that as many people as possible should be able to work from home, even without obligation. Potentially, $34 \%$ of jobs could be accomplished at home, which implies reduced mobility by nearly the same percentage. Potentially, the younger population could be the first to be released from the lockdown to further alleviate any subsequent stress on the health system and potentially reinforce the collective immunity effect. ${ }^{1,2}$

The results derived from the proposed model can provide useful information on maintaining/releasing containment and avoiding adverse effects in any country. This model is sensitive to health interventions and decisions made, which can lead to variations in predictions. Thus, nations can rely on official databases to reveal biases in case confirmation and information.

\section{Conclusion and Recommendation}

The findings emphasize that gradual, cautious steps should be taken with the ease in restrictions to save resources and lives. The work of primary care and nursing staff is crucial in the post-peak COVID-19 phase. Lastly, long-term preparations should be made to contain future waves of new cases.

\begin{abstract}
Abbreviations
COVID-19: coronavirus infection disease; PCR test: polymerase chain reaction test; MAD: mean absolute deviation; MSD: mean signed deviation; MAPE: mean absolute percentage error

\section{Ethics Approval and Consent to Participate}

Data are public and available on the Chilean Ministry of Science, Technology, Knowledge, and Innovation website. No committee approval was required because the study adheres to ethical aspects.
\end{abstract}

\section{Competing Interest}

The authors declare that there are no significant competing financial, professional, or personal interests that might have affected the performance.

\section{Availability of Data and Materials}

The data are available on the website of the Ministry of Science, Technology, Knowledge, and Innovation of Chile (http://www.minciencia.gob.cl/covid19) through the open data of the COVID-19 Database.

\begin{abstract}
Authors' Contribution
DS-M-R and AC-N: concept and design of the article, collecting of results, analysing and interpretating data, writing the article, critical review of the article, approving the final version, contributing of patients or study material, statistical advice, and technical or administrative advice. FR-L: concept and design of the article, collection of results, writing of the article, critical review of the article, approval of the final version, the contribution of patients or study material, and technical or administrative advice. PS-M-R: concept and design of the article, collection of results, analysis, and interpretation of data, writing of the article, critical review of the article, approval of the final version, the contribution of patients or study material, and technical or administrative advice. PD-C: conception and design of the article, collection of results, analysis, and interpretation of data, writing of the article, critical review of the article, approval of the final version, statistical advice, and technical or administrative advice. VPD-N: concept and design of the article, collection of results, analysis and interpretation of data, writing of the article, critical review of the article, approval of the final version, and statistical advice.
\end{abstract}

\section{Acknowledgment}

Not Applicable

\section{Additional Information}

David San-Martín-Roldán ${ }^{1}$, Francisca Rojo-Lazo ${ }^{1}$, Aracelis CalzadillaNúñez ${ }^{2}$, Pablo San-Martín-Roldán ${ }^{3}$, Patricia Díaz-Calzadilla ${ }^{4}$, Víctor P Díaz-Narváez ${ }^{5}$

${ }^{1}$ Escuela de Obstetricia y Puericultura, Facultad de Medicina, Universidad de Valparaíso, Valparaíso, Chile; ${ }^{2}$ Facultad de Ciencias de la Salud, Universidad Autónoma de Chile, Santiago, Chile; ${ }^{3}$ Instituto 
de Nutrición y Tecnología de los Alimentos (INTA), Universidad de Chile, Santiago, Chile; ${ }^{4}$ Estudiante de Medicina. Facultad de Ciencias de la Salud. Universidad Autónoma de Chile. Santiago, Chile; ${ }^{5}$ Facultad de Odontología. Universidad Andres Bello. Santiago. Chile.

\section{References}

1. Rawson T, Brewer T, Veltcheva D, Huntingford C, Bonsall MB. How and when to end the COVID-19 lockdown: an optimization approach. Frontiers in Public Health 2020; 8: 262.

2. Ng WL. To lockdown? When to peak? Will there be an end? A macroeconomic analysis on COVID-19 epidemic in the United States. Journal of Macroeconomics 2020; 65: 103230.

3. Adhikari R SP, Meng S, Wu YJ, et al. Epidemiology, causes, clinical manifestation and diagnosis, prevention and control of coronavirus disease [COVID-19] during the early outbreak period: a scoping review. Infectious Diseases of Poverty. 2020; 9 (1): 29.

4. Confirmed Cases by country/Region/Sovereignty. COVID-19 dashboard by the center for systems science and engineering (CSSE) at Johns Hopkins University; 2020.

5. Basu D, Salvatore M, Ray D, Kleinsasser M, Purkayastha S, Bhattacharyya R, et al. A comprehensive public health evaluation of lockdown as a non-pharmaceutical intervention on COVID-19 spread in India: national trends masking state-level variations. medRxiv: The Preprint Server for Health Sciences. 2020.

6. Nicola M, Alsafi Z, Sohrabi C, Kerwan A, Al-Jabir A, Iosifidis C, et al. The socio-economic implications of the coronavirus pandemic [COVID-19]: a review. International Journal of Surgery 2020; 78: 185-93.

7. Dubey S, Biswas P, Ghosh R, Chatterjee S, Dubey MJ, Chatterjee S, et al. Psychosocial impact of COVID-19. Diabetes \& Metabolic Syndrome. 2020; 14 (5): 779-88.

8. Base de Datos C-19. Ministerio de ciencia, tecnología, conocimiento e innovación de Chile; 2020.

9. Díaz-Narváez V, et al. Which curve provides the best explanation of the growth in confirmed COVID-19 cases in Chile? Revista LatinoAmericana de Enfermagem 2020; 28: e3346.

10. Sjödin H, Wilder-Smith A, Osman S, Farooq Z, Rocklöv J. Only strict quarantine measures can curb the coronavirus disease (COVID-19) outbreak in Italy, 2020. Eurosurveillance. 2020; 25 (13): 2000280

11. COVID-19 in Germany; 2020.

12. de Covid S - 19 En españa. Ministerio de Sanidad 2020; 2020.
13. Infection Au nouveau coronavirus [SARS-CoV-2], COVID-19, France et Monde. Santé Publique France 2020; 2020.

14. COVID-19: situation report update at 5 Jul 2020; 2020.

15. Current information about COVID-19 (novel coronavirus). Rijksinstituut voor Volksgezondheid en Milieu 2020; 2020.

16. Department of Health and Social Care and Public Health England. Number of coronavirus (COVID-19) cases and risk in the UK; 2020.

17. Federal Ministry Republic of Austria Social Affairs, Health, Care and Consumer Protection. Official c - 19 dashboard-explanatory notes; 2020.

18. Website of the Republic of Poland. Coronavirus: information and recommendations; 2020.

19. de Dados D, da Saúde D-G. Ministério Da Saúde; 2020.

20. New coronavirus; 2020.

21. National Health Commission of the PRC. Daily briefing; 2020.

22. Cases in Korea. Coronavirus Disease-19, Republic of Korea 2020; 2020.

23. Australian Government Department of Health. Coronavirus (COVID19) at a glance infographic collection; 2020.

24. COVID-19 (novel coronavirus). Ministry of Health Manatū Hauora 2020; 2020.

25. Plan Nacional coronavirus. Ministerio de Salud Pública; 2020.

26. Oxford Martin School, University of Oxford. Policy responses to the coronavirus pandemic: statistics and research. Our world in data; 2020.

27. Garg S, Basu S, Rustagi R, Borle A. Primary health care facility preparedness for outpatient service provision during the COVID-19 pandemic in India: cross-sectional study. JMIR Public Health and Surveillance. 2020; 6 (2): e19927.

28. Kwok KO, Florence L, Wan In W, Samuek YSW, Julian WTT. Herd immunity - estimating the level required to halt the COVID-19 epidemics in affected countries. Journal of Infection. 2020 Jun; 80 (6): e32-3.

29. Zhongjie L, Qiulan C, Luzhao F, Rodewald L, Yinyin X, Hailiang Y, et al. China CDC COVID-19 emergency response strategy team. Active case finding with case management: the key to tackling the COVID-19 pandemic. Lancet. 2020; 396 (10243): 63-70.

30. Saez M, Tobias A, Varga D, Barceló MA. Effectiveness of the measures to flatten the epidemic curve of COVID-19. The case of Spain. Science of the Total Environment. 2020; 727: 138761. 